\title{
Correction to: Effects of early feeding on growth velocity and overweight/obesity in a cohort of HIV unexposed South African infants and children
}

Vundli Ramokolo $0^{1,2^{*}}$, Carl Lombard ${ }^{3}$, Meera Chhagan ${ }^{2,4,5^{\wedge}}$, Ingunn M. S. Engebretsen², Tanya Doherty ${ }^{1,4}$, Ameena E. Goga ${ }^{1,7}$, Lars Thore Fadnes ${ }^{2,6}$, Wanga Zembe ${ }^{1}$, Debra J. Jackson ${ }^{4}$ and Jan Van den Broeck ${ }^{2 *}$

\section{Correction}

After publication of this article [1] it was brought to our attention that there were errors in the text under the heading 'Data cleaning', and in Table 3. The corrected text and updated Table 3 are given in this erratum.

"Anthropometric measurement values and Z-scores were flagged for verification if any of the following criteria were met: WAZ $<-6$ or $>5$, WLZ $<-5$ or $>5, \mathrm{LAZ}<-6$ or $>6$, WLZ $>3$ and LAZ $<-3$; 2) extreme changes in LAZ and WLZ (greater than 2.5 or 3 ) between consecutive visits; 3 ) BMI-for-age Z-score $\geq 6$. All the flagged anthropometric observations were assessed and values treated as missing if no plausible explanation was determined."

Table 3 Period-1 (the 3/6 week-12 week) and period-2 (12-24 weeks) mean weight velocity (WVZ) and length velocity (LVZ) by infant feeding ${ }^{a}$

\section{3 week feeding}

Period 1: 3/6-12 weeks

Never breastfed

Breastfed $^{c}$

WZP1 $(N=522)$

n mean $\pm S D$

$n$

mean \pm SD

P-value

LVZP1 $(N=402)$

$60 \quad 1.58 \pm 1.72$

462

$0.99 \pm 1.60$

46

$1.69 \pm 2.62$

356

$1.37 \pm 2.44$

12 week feeding

Period 2: 12-24 weeks

Never breastfed

Breastfed $^{c}$

WZP2 $(N=494)$

n mean $\pm S D$

n

mean $\pm S D$

$98 \quad 1.07 \pm 1.75$

394

$0.64 \pm 1.57$

LVZP2 (N = 477)

$93 \quad 0.82 \pm 2.62$

Values are mean + SD of velocity Z-scores based on WHO standard. LVZ length

velocity $Z$-score, $P 1$ Period, $P 2$ Period-2, $W V Z$ weight velocity $Z$-score

${ }^{\mathrm{b}}$ Student test $P$ values for group comparisons a $5 \%$ significance level

'Children received breast milk in addition to other solids and liquids

* Correspondence: vundli.ramokolo@mrc.ac.za

Deceased

${ }^{1}$ Health Systems Research Unit, South African Medical Research Council, Cape Town, South Africa

${ }^{2}$ Centre for International Health, Department of Global Public Health and Primary Care, University of Bergen, Bergen, Norway

\section{Author details}

${ }^{1}$ Health Systems Research Unit, South African Medical Research Council, Cape Town, South Africa. ${ }^{2}$ Centre for International Health, Department of Global Public Health and Primary Care, University of Bergen, Bergen, Norway. ${ }^{3}$ Biostatistics Unit, South African Medical Research Council, Cape Town, South Africa. ${ }^{4}$ School of Public Health, University of the Western Cape, Cape Town, South Africa. ${ }^{5}$ Department of Pediatrics, University of KwaZulu Natal, KwaZulu Natal, South Africa. ${ }^{6}$ Department of Clinical Dentistry, University of Bergen, Bergen, Norway. ${ }^{7}$ Department of Paediatrics and Child Health, Kalafong Hospital, University of Pretoria, Pretoria, South Africa.

Received: 30 October 2017 Accepted: 2 November 2017

Published online: 13 November 2017

\section{Reference}

1. Ramokolo V, et al. Effects of early feeding on growth velocity and overweight/ obesity in a cohort of HIV unexposed South African infants and children. Int Breastfeed J. 2015;10:14. https://doi.org/10.1186/s13006-015-0041-x.

\section{$\int$ Biomed Central}

(c) The Author(s). 2017 Open Access This article is distributed under the terms of the Creative Commons Attribution 4.0 International License (http://creativecommons.org/licenses/by/4.0/), which permits unrestricted use, distribution, and reproduction in any medium, provided you give appropriate credit to the original author(s) and the source, provide a link to the Creative Commons license, and indicate if changes were made. The Creative Commons Public Domain Dedication waiver (http://creativecommons.org/publicdomain/zero/1.0/) applies to the data made available in this article, unless otherwise stated. 